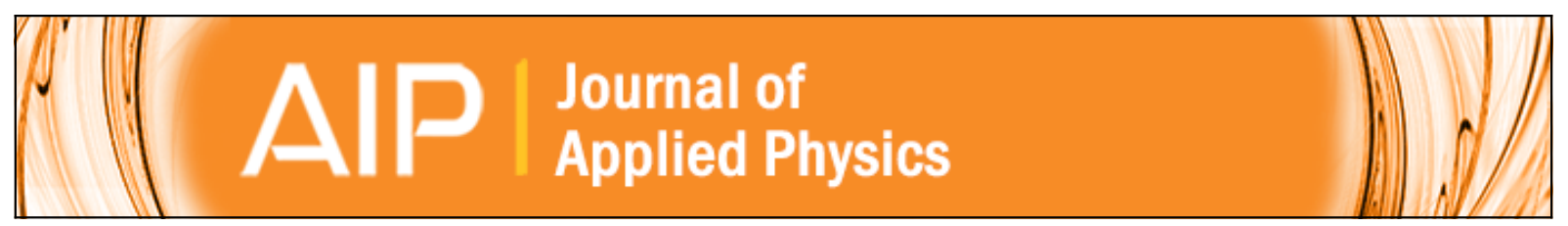

\title{
Self-excited vibrational viscometer for high-viscosity sensing
}

Hiroshi Yabuno, Keiichi Higashino, Masaharu Kuroda, and Yasuyuki Yamamoto

Citation: Journal of Applied Physics 116, 124305 (2014); doi: 10.1063/1.4896487

View online: http://dx.doi.org/10.1063/1.4896487

View Table of Contents: http://scitation.aip.org/content/aip/journal/jap/116/12?ver=pdfcov

Published by the AIP Publishing

\section{Articles you may be interested in}

A low cycle fatigue test device for micro-cantilevers based on self-excited vibration principle

Rev. Sci. Instrum. 85, 105005 (2014); 10.1063/1.4898668

Self-excited coupled cantilevers for mass sensing in viscous measurement environments

Appl. Phys. Lett. 103, 063104 (2013); 10.1063/1.4817979

Design of a high precision falling-ball viscometer

Rev. Sci. Instrum. 76, 025109 (2005); 10.1063/1.1851471

Low-shear-rate capillary viscometer for polymer solution intrinsic viscosity determination at varying temperatures

Rev. Sci. Instrum. 74, 176 (2003); 10.1063/1.1529302

The Effects of Temperature and Pressure on the Dynamic Longitudinal Volume Viscosity of Two Model Polymers J. Rheol. 32, 533 (1988); 10.1122/1.550000

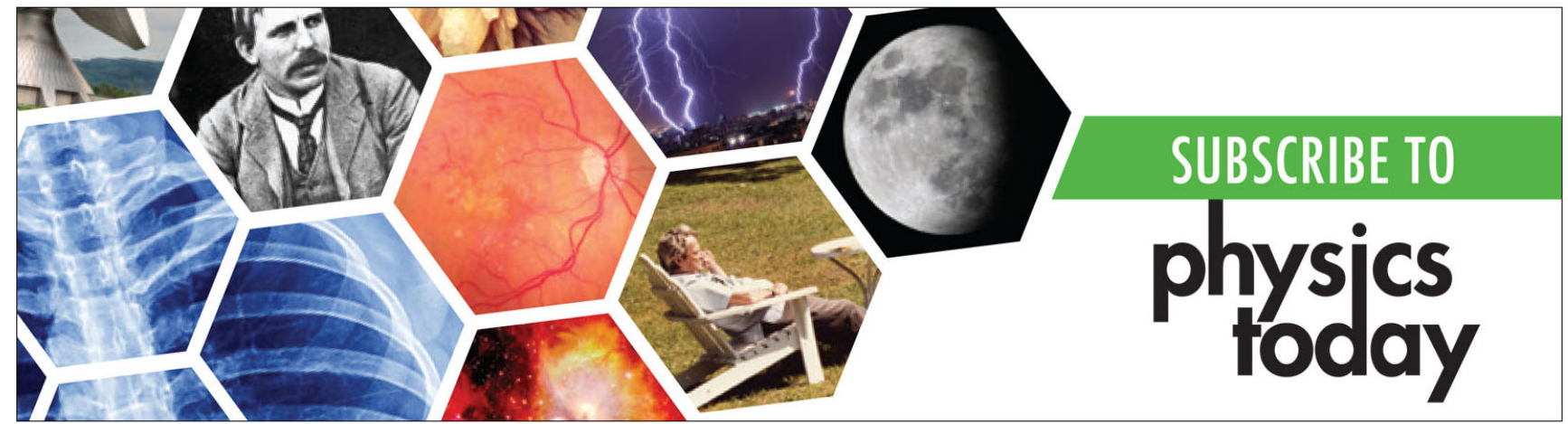




\title{
Self-excited vibrational viscometer for high-viscosity sensing
}

\author{
Hiroshi Yabuno, ${ }^{1}$ Keiichi Higashino, ${ }^{2}$ Masaharu Kuroda, ${ }^{3}$ and Yasuyuki Yamamoto ${ }^{4}$ \\ ${ }^{1}$ Graduate School of Systems and Information Engineering, University of Tsukuba, \\ Tsukuba Science City 305-8573, Japan \\ ${ }^{2}$ Faculty of Science and Technology, Keio University, Kohoku, Yokohama 223-8522, Japan \\ ${ }^{3}$ Graduate School of Engineering, University of Hyogo, Himeji, Hyogo 671-2201, Japan \\ ${ }^{4}$ National Institute of Advanced Industrial Science and Technology (AIST), Tsukuba Science City 305-8568, \\ Japan
}

(Received 20 July 2014; accepted 15 September 2014; published online 24 September 2014)

\begin{abstract}
A method for vibrational viscometers capable of high-viscosity measurements using self-excited oscillations is proposed and assessed both theoretically and experimentally. Such viscometers are well-known for their rapid response and miniaturization. Unlike conventional methods based on $Q$-value estimations obtained experimentally from the frequency response or resonance curve, we describe the use of self-excited oscillations in viscosity measurements using positive velocity feedback control without relying on the frequency response curve. Such measurements become possible even for high viscosities where the peak of the frequency response curve is ambiguous or does not exist, i.e., the $Q$-value cannot be estimated from such curves. Furthermore, the validity of the proposed method is experimentally tested using a prototype self-excited viscometer. Downsized oscillators such as micro- or nanoscale cantilevers can be self-excited following a straightforward application of the method. They are expected to enable not only localized monitoring of changes in high viscosity with time but also spatial high-viscosity measurements by the distributed arrangement of the devices. (C) 2014 AIP Publishing LLC.
\end{abstract}

[http://dx.doi.org/10.1063/1.4896487]

\section{INTRODUCTION}

Vibrating-type viscometers ${ }^{1,2}$ are receiving increasing attention for their rapid response, which allows for instantaneous and continuous measurements of changes in the viscosity of the sample liquid occurring with time, i.e., on-line monitoring. The methods of measurement based on vibrations are easily miniaturized using such devices as micro-cantilevers and micro-fibers. ${ }^{3-6}$ These devices enable not only localized monitoring of the viscosity in a liquid sample but also spatial measurements using a distributed arrangement of the devices. The measurement procedure of conventional vibrating-type viscometers is to use the frequency response or resonance curve under external or forced excitation. The frequency response curve of the viscometer has different kurtosis at the resonance peak depending on the amount of viscous force acting on the vibrational part immersed in the liquid sample. Hence, using Q-values estimated from the half band width of the experimentally obtained frequency response curve, the viscosity can be estimated (for example, see Ref. 7). However, in the high viscosity range, it is very difficult to accurately estimate differences in Q-values because of the widening of the half band and the shifting of the resonance peak in the frequency response curve. ${ }^{8}$ Moreover, for much higher viscosities where resonance peaks do not appear in the frequency response curves, methods involving external excitations are not applicable in determining the viscosity. The use of frequency-phase characteristics, ${ }^{9,10}$ the shift of the resonance frequency, ${ }^{11}$ and nonlinear phenomena such as superharmonic resonance ${ }^{12}$ have been proposed, but these methods encounter the same issue as conventional methods based on the $Q$-value or may have low accuracy in the highviscosity regime.

Such phenomena as parametric resonance and selfexcited oscillation whose resonance mechanisms are essentially different from that for harmonic resonance under conventional external excitation, can be used to enhance the performance of resonators used in atomic force microscopy, ${ }^{13,14}$ mechanical filters, ${ }^{15}$ and mass sensors. ${ }^{16}$

In the present paper, we propose a method based on self-excited oscillations to make high viscosity measurements without the need to obtain frequency response curves of external excitations. A positive velocity feedback is applied to produce the self-excited oscillation in the vibrational part immersed in the liquid sample. The feedback force can compensate for the energy dissipation ${ }^{17}$ in the motion of a disk immersed in the liquid sample caused by viscous action acting on the disk. When the feedback gain exceeds a critical value, the feedback force changes the damping characteristics of the disk motion from positive to negative damping ${ }^{18}$ and the disk oscillates producing the self-excitations in the liquid sample. The critical feedback gain depends on the strength of the viscous damping force acting on the disk and the viscosity of the sample liquid is estimated from the critical feedback gain. Therefore, the proposed method requires no frequency response curve and clarification from theory shows that viscosity can be measured irrespective of value. In this paper, experimental confirmation is given of very high viscosity measurements using a prototype self-excited viscometer. 


\section{VISCOSITY MEASUREMENT BASED ON SELF-EXCITED OSCILLATIONS}

\section{A. Mathematical model and equation of motion}

We consider the analytical model shown in Fig. 1. As in conventional vibrational viscometers, the thin rigid disk suspended by the spring can laterally oscillate due to the restoring force. The motion can be identified with that of a mass-spring system with mass $m$ and spring stiffness $k$. When the disk is immersed in the liquid sample, the fluid force acting on the disk affects the motion of the disk. An actuator is also attached for lateral excitations; in this study, an applied force is used to produce self-excited oscillations in accordance with a method described below.

In the derivation of the equation of motion for the disk, the conventional expression ${ }^{19}$ for the viscous fluid force, $F_{f}$, acting on the disk from the sample liquid is used. However, the resulting equation of motion for the proposed viscometer is significantly different from that for conventional vibrational viscometers because of differences in their excitation mechanisms. The equation of motion for conventional methods based on external sinusoidal excitations is nonhomogeneous, whereas for our proposed method based on positive-velocity feedback it is homogeneous. Also in the theoretical analysis for the equation of motion, a different approach, i.e., stability analysis of the trivial steady state, has to be used in the proposed method.

First, the fluid velocity in the $x$-direction, $v_{x}(y, t)$, is governed by

$$
\frac{\partial v_{x}}{\partial t}=\frac{\eta}{\rho} \frac{\partial^{2} v_{x}}{\partial y^{2}}
$$

where the origin of the Cartesian coordinate system $x-y$ is set at a point on the surface of the disk in the static equilibrium state; the $x$-axis aligns with the applied forces, as depicted in Fig. 1, with the $y$-axis directed outward from the page. The coefficients $\eta$ and $\rho$ are the respective dynamic viscosity and fluid density of the liquid. The boundary conditions at $y=\infty$ and $y=0$ are, respectively, $v_{x}(\infty, t)=0$ and

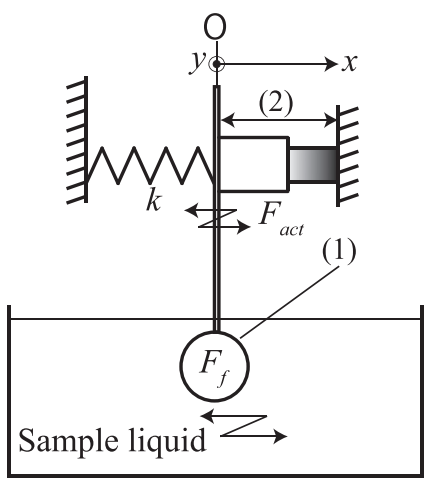

FIG. 1. Analytical model of self-excited vibrational viscometer for highviscosity measurement corresponding to the experimental apparatus shown in Fig. 2, where (1) is a rigid vibrational disk immersed in a liquid sample and (2) is a linear motor for positive velocity feedback control to produce the self-excited oscillation. The stiffness with respect to the motion of the disk along the $x$-axis is denoted $k$.

$$
v_{x}(0, t)=v_{a} \exp (j \omega t),
$$

where $j^{2}=-1$, and $v_{a}$ and $\omega$ are, respectively, the complex amplitude and the frequency of the velocity of the disk. Unlike conventional methods, $\omega$ is unknown at this stage and is determined later from the balance of the total forces acting on the disk.

Setting

$$
v_{x}(y, t)=g(y) \exp (j \omega t)
$$

yields

$$
\frac{d^{2} g}{d y^{2}}-j \frac{\rho \omega}{\eta} g=0
$$

With the above boundary conditions, $v_{x}$ is expressed as

$$
v_{x}(y, t)=v_{a} \exp \left\{-\sqrt{\frac{\rho \omega}{2 \eta}}(1+j) y\right\} \exp (j \omega t) .
$$

Then, the fluid force $F_{f}$ along the $x$-axis is

$$
F_{f}=\left.S \eta \frac{\partial v_{x}}{\partial y}\right|_{y=0}=-S \sqrt{\frac{\rho \eta \omega}{2}} v_{a}(1+j) \exp (j \omega t),
$$

where $S$ is the area of the vibrational disk immersed in the liquid. This equation is derived from the fluid force, which acts per unit area on a plate of finite extent, and does not include the additional fluid force induced at the edges. ${ }^{19}$ The influence of these edges on the proposed viscosity measurement method is discussed in relation to experimental data in Sec. III C.

Invoking Newton's second law, we obtain the equation of motion for the disk

$$
m \frac{d^{2} x}{d t^{2}}+k x=F_{f}+F_{a c t},
$$

where the left-hand side is the sum of the inertial and abovementioned spring forces, and the right-hand side is the sum of the fluid force $F_{f}$ received from the sample liquid and the force $F_{a c t}$ applied by an actuator to produce the self-excited oscillations.

\section{B. Self-excited oscillation under positive velocity feedback and high-viscosity measurement}

To move the disk through the self-excitation, we set the applied force using feedback determined by the disk's velocity $v_{x}(0, t)$

$$
F_{a c t}=C v_{x}(0, t),
$$

where $C(>0)$ is the positive feedback gain. Because the frequency component in the solution for $x$, i.e., the response frequency of the disk, is equal to that in $F_{a c t}$ due to the linearity of Eq. (7) under this feedback, the displacement of the disk assumes the following form:

$$
x=a(t) \exp (j \omega t),
$$

where $a$ and $\omega$ are, respectively, the real-valued response amplitude and the real-valued response frequency equal to 
the frequency of the velocity of the disk assumed in advance in Eq. (2). The velocity and acceleration of the disk are expressed using the dimensionless amplitude $a^{*}(=a / D$, where $D$ is the diameter of the disk) and the dimensionless time $t^{*}(=t /(1 / \Omega))$ as

$$
\begin{gathered}
\frac{d^{2} x}{d t^{2}}=D \Omega^{2}\left(\frac{d^{2} a^{*}}{d t^{* 2}}+2 j \omega^{*} \frac{d a^{*}}{d t^{*}}-\omega^{* 2} a^{*}\right) \exp \left(j \omega^{*} t^{*}\right), \\
\frac{d x}{d t}=D \Omega\left(\frac{d a^{*}}{d t^{*}}+j \omega^{*} a^{*}\right) \exp \left(j \omega^{*} t^{*}\right),
\end{gathered}
$$

where $D$ and $1 / \Omega=\sqrt{m / k}$ are the representative length and time, respectively, and $\omega^{*}=\omega / \Omega$. Also, the complex amplitude of the velocity is

$$
v_{a}=D \Omega\left(\frac{d a^{*}}{d t^{*}}+j \omega^{*} a^{*}\right) .
$$

We substitute Eqs. (10) and (11) into Eq. (7), and take into account Eq. (12). Then, separating Eq. (7) into real and imaginary parts yields

$$
\begin{gathered}
\frac{d^{2} a^{*}}{d t^{* 2}}+\left(Q^{*}-C^{*}\right) \frac{d a^{*}}{d t^{*}}+\left(1-\omega^{* 2}-\omega^{*} Q^{*}\right) a^{*}=0, \\
\frac{d a^{*}}{d t^{*}}+\frac{\omega^{*}}{2 \omega^{*}+Q^{*}}\left(Q^{*}-C^{*}\right) a^{*}=0,
\end{gathered}
$$

where $C^{*}$ and $Q^{*}$ are dimensionless parameters defined as

$$
C^{*}=\frac{C}{m \Omega}, \quad Q^{*}=\frac{S}{m \Omega} \sqrt{\frac{\rho \eta \omega}{2}} .
$$

Note $C^{*}$ is the dimensionless feedback gain. The imaginary part Eq. (14) of the equation of motion consists of viscous damping and velocity feedback terms because these terms include the first time derivative and their phase leads $\pi / 2$ to the displacement. Therefore, the time variation of the amplitude governed by these terms is calculated from the imaginary part Eq. (14). In contrast, the real part Eq. (13) of the equation of motion consists of the inertia terms, whose phase yields a $\pi$ shift, and the stiffness term giving no phase shift to the displacement and determines the response frequency as simple spring-mass systems.

Here, we consider the case when the dimensionless feedback gain $C^{*}$ is set near $Q^{*}$. We explicitly express the near equality using the order parameter $\epsilon(|\epsilon| \ll 1)$ as follows:

$$
P=C^{*}-Q^{*}=\epsilon \hat{P}(\hat{P}=O(1)) .
$$
leads to

Then, using the slow time scale $t_{1}\left(=\epsilon t^{*}\right),{ }^{20}$ Eq. (14)

$$
\frac{d a^{*}}{d t_{1}}-\frac{\omega^{*} \hat{P}}{2 \omega^{*}+Q^{*}} a^{*}=0 .
$$

As a result, $a^{*}$ is a function of $t_{1}$ and its time variation can be regarded to be very slow. Thus, considering $a^{*}$ is a function of $t_{1}$, we can rewrite Eq. (13) as

$$
\epsilon^{2} \frac{d^{2} a^{*}}{d t_{1}^{2}}+\epsilon^{2} \hat{P} \frac{d a^{*}}{d t_{1}}+\left(1-\omega^{* 2}-\omega^{*} Q^{*}\right) a^{*}=0 .
$$
yields

Neglecting the terms in $\mathrm{O}\left(\epsilon^{2}\right)$ and considering $a^{*} \neq 0$

$$
1-\omega^{* 2}-\omega^{*} Q^{*}=0 .
$$

Let us examine the stability of the disk plate motion using the time variation of the amplitude. The solution to Eq. (14) or (16) is

$$
a^{*}\left(t^{*}\right)=a_{0}^{*} \exp \left\{\frac{\omega^{*} P}{2 \omega^{*}+Q^{*}} t^{*}\right\},
$$

where $a_{0}^{*}$ is a dimensionless constant determined by the initial displacement. The solution can be written in the dimensional form as

$$
a(t)=a_{0} \exp \left\{\frac{\omega\left(C-S \sqrt{\frac{\rho \eta \omega}{2}}\right)}{2 m \omega+S \sqrt{\frac{\rho \eta \omega}{2}}} t\right\},
$$

where $a_{0}=D a_{0}^{*}$. The change in stability occurs when the feedback gain $C$ is $C_{c r}$, where

$$
C_{c r}=S \sqrt{\frac{\rho \eta \omega}{2}}
$$

and $C_{c r}$ is hereafter called the critical feedback gain. Hence, the so-called Hopf bifurcation ${ }^{21}$ occurs at $C=C_{c r}$. If $C<C_{c r}$, the disk is stable and applying the initial disturbance, the response amplitude decays with time because the feedback control force is less than the damping force due to the fluid force acting on the disk, i.e., $S \sqrt{\frac{\rho \eta \omega}{2}} v_{a}$. In contrast, for $C>C_{c r}$, because the feedback control force is higher than the damping force, the disk undergoes a self-excited oscillation through Hopf bifurcation and the response amplitude grows with time.

We propose using the sudden change in stability, which depends on the relationship between the feedback gain $C$ and the viscosity of the liquid sample. Rearranging Eq. (20), the viscosity $\eta$ is written

$$
\eta=\frac{2}{\rho \omega}\left(\frac{C_{c r}}{S}\right)^{2}
$$

where the values of $S$ and $\rho$ are known in advance and the critical feedback gain $C_{c r}$ and $\omega$ are experimentally determined as described in the following. In the experiment, we gradually increase the feedback gain $C$ from a low value. The occurrence of the self-excited oscillation means that $C$ exceeds $C_{c r}$. The experimentally obtained value $C$ at the stability boundary corresponds to $C_{c r}$.

From the above discussion, the response frequency $\omega$ is found by Eq. (17). The dimensional form is expressed as

$$
m \omega^{2}-k+S \omega \sqrt{\frac{\rho \eta \omega}{2}}=0 .
$$


Therefore, if the feedback gain $C$ is slightly above $C_{c r}$, i.e., $0<P \ll 1$ in Eq. (15), theory clarifies that the response frequency $\omega$ is independent of the time-dependent response amplitude in the self-excited oscillation. The response frequency obtained from the experimental time history of the self-excited disk can be used as $\omega$ in Eq. (21). A more practical measurement strategy will be given later together with experimental results.

\section{EXPERIMENTS}

\section{A. Measurement method}

To confirm the validity of our method for high-viscosity measurements, we conducted experiments using a prototype self-excited viscometer (Fig. 2); the mass, consisting mainly of the disk and the mover of the linear motor, is $m=1.6 \mathrm{~kg}$, and the diameter and width of the disk are $80 \mathrm{~mm}$ and $1 \mathrm{~mm}$, respectively. The block diagram of the practical measurement system is shown in Fig. 3.

The displacement of the disk is measured by a laser displacement sensor (Keyence Corp.: LB-300) whose gain is $G_{S}[\mathrm{~V} / \mathrm{m}]$. The signal is differentiated by an analog differentiator whose gain is $G_{D}[1 / \mathrm{s}]$ and is multiplied by the variable control gain $G$ in an analog circuit. Furthermore, the current proportional to the resulting voltage is produced by a bipolar power supply (Kikusui Corp.: PBX40-10) whose gain is $G_{P}[\mathrm{~A} / \mathrm{V}]$. The current to the linear motor (Showa Densen Corp.: R26, the thrust constant is $G_{A}=2 \mathrm{~N} / \mathrm{A}$ ) supplies a thrust proportional to the current in the disk. The gains, except for $G$, are constant and their actual values are not used in subsequent viscosity measurements because the viscosity of the reference or calibration liquid is employed. The theoretical feedback gain $C$ in Eq. (8) corresponds to $G_{S} G_{P} G_{A} G / G_{D} ; C$ is experimentally changed by setting the variable gain $G$ in the analog circuit. The value of $G$ corresponding to the critical feedback gain $C_{c r}$ is expressed as $G=C_{c r} G_{D} /\left(G_{S} G_{P} G_{A}\right) \equiv G_{c r}$.

By gradually increasing $G$ above $G_{c r}$, the disk destabilizes and oscillates in the liquid sample through

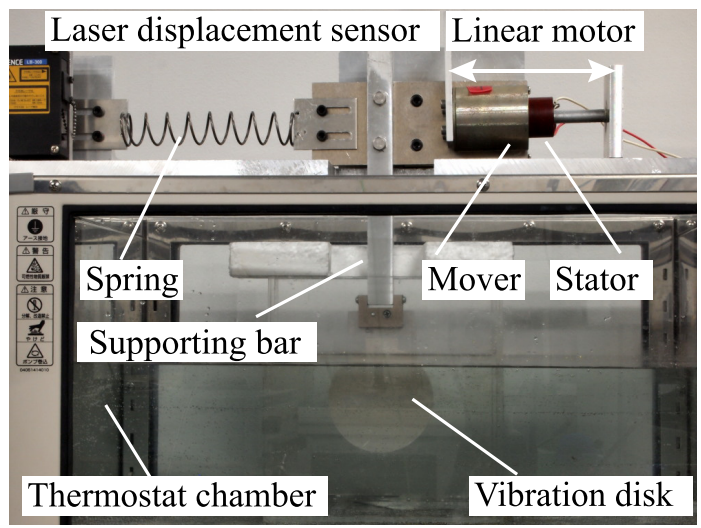

FIG. 2. Experimental setup of a self-excited vibrating-type viscometer for high-viscosity measurements. The oscillator consists of a spring, linear motor, and a bar connected to the disk immersed in the liquid sample. The linear motor produces the positive velocity feedback force in accordance with the theoretically proposed model; the corresponding block diagram is shown in Fig. 3.

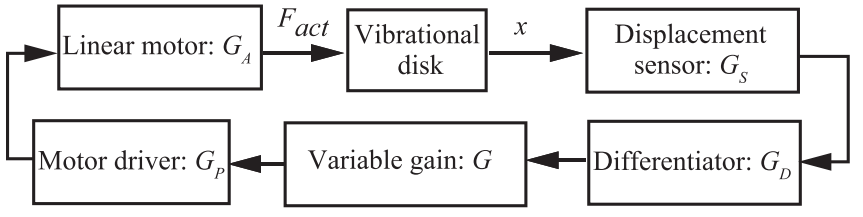

FIG. 3. Feedback loop producing the self-excited oscillations ( $x$ : displacement of disk, $F_{a c t}$ : feedback control force). $F_{\text {act }}$ is applied to the disk in accordance with the proposed positive velocity feedback. Increasing the variable gain $G$ and exceeding the critical gain $G_{c r}$ produces self-excited oscillations.

self-excitation. Therefore, by the experimentally obtained value $G=G_{c r}$ at the stability boundary and response frequency $\omega, \eta$ in Eq. (21) can be expressed as

$$
\eta=\frac{2}{\rho \omega}\left(\frac{G_{S} G_{P} G_{A} G_{c r}}{S G_{D}}\right)^{2} .
$$

We tried high-viscosity measurements using samples of a standard liquid made of hydrocarbon to calibrate the viscometers (Nippon Grease Corp.: JS160000), the accuracy of which comply with Japanese Industrial Standard (JIS Z 8809); labeling the samples by (i)-(vii), the viscosity $\eta$ of each depends on temperature, as listed in Table I, and were determined by setting the thermostat of the chamber to temperature $T$ of the standard liquid.

\section{B. Self-excited oscillation in high-viscosity liquid}

We began with the conventional measurement method applying an external excitation to the high-viscosity liquid (i), setting $F_{a c t}=F_{a} \cos \nu t$ without feedback (see Eq. (7)). Figure 4 shows the experimentally obtained frequency response curve under $F_{a}=0.8 \mathrm{~N}$, where $x_{a m p}$ is the response amplitude depending on the excitation frequency $\nu$. Because there is no peak due to the high viscosity of the liquid, conventional methods do not apply as these are based on the $Q$ factor estimated from the half band width of the frequency response curve.

Using liquid (ii), we investigated the validity of the proposed method by the dynamic responses under an initial disturbance. Without feedback, $G=0$, the dynamic response falls within the over-damped regime (Fig. 5(a)). By increasing the gain of $G$, the disk undergoes damped oscillatory motion (Fig. 5(b)) because the energy dissipation in the motion due to the liquid's high viscosity decreases by the positive velocity feedback-control force. Increasing the gain further, we observe the self-excited oscillation of the disk (Fig. 5(c)). These oscillations indicate that the feedback

TABLE I. Properties of the liquid samples and measured viscosities.

\begin{tabular}{lccccccc}
\hline \hline Label of liquids & (i) & (ii) & (iii) & (iv) & (v) & (vi) & (vii) \\
\hline$T\left({ }^{\circ} \mathrm{C}\right)$ & 20.2 & 21.0 & 21.6 & 22.3 & 22.8 & 24 & 24.7 \\
$\rho\left(\times 10^{2} \mathrm{~kg} / \mathrm{m}^{3}\right)$ & 8.95 & 8.95 & 8.95 & 8.94 & 8.94 & 8.93 & 8.93 \\
$\eta(\mathrm{Pa} \mathrm{s})$ & 141 & 130 & 122 & 114 & 109 & 96.8 & 90.5 \\
$\eta_{a}(\mathrm{~Pa} \mathrm{~s})$ & $\mathrm{NA}$ & 134 & 118 & 110 & 100 & 87.6 & 72.9 \\
$\eta_{\sigma}(\mathrm{Pa} \mathrm{s})$ & $\mathrm{NA}$ & 2.9 & 2.8 & 2.7 & 1.1 & 2.1 & 0.9 \\
Error $(\%)$ & $\mathrm{NA}$ & 2.9 & 3.6 & 3.8 & 8.1 & 9.5 & 19 \\
\hline \hline
\end{tabular}




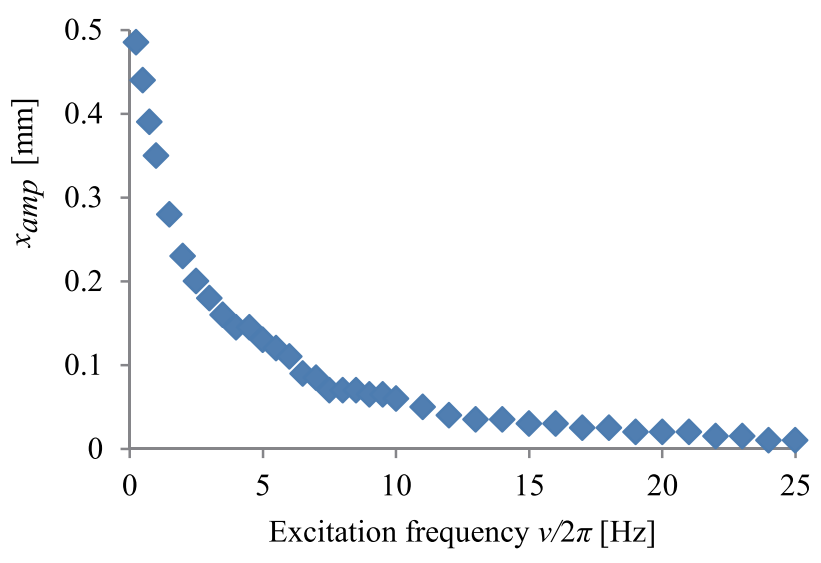

FIG. 4. Experimental frequency response curve obtained by the conventional method based on external excitation $F_{a c t}=F_{a} \cos \nu t ; F_{a}=0.8 \mathrm{~N}$. No peak appears as the test liquid has high viscosity $\left(1.41 \times 10^{2} \mathrm{~Pa} \mathrm{~s}\right)$, and hence viscosity cannot be experimentally estimated.

control force with sufficient gain of $G$ compensates the energy dissipation and changes the damped system from positive to negative. In each experiment, we applied an initial single square wave-shaped disturbance of magnitude and duration $2 \mathrm{~N}$ and $0.1 \mathrm{~s}$, respectively. Note that, if the artificial disturbance is not applied, self-excited oscillations cannot be produced because of inherent Coulomb friction in the mechanical moving parts, which is not taken into account in the analysis of Eq. (7); this effect on the self-excited oscillation is theoretically and experimentally clarified in detail in the study. ${ }^{22}$

\section{High-viscosity measurements}

Furthermore, to investigate the quantitative validity of the proposed method, we estimate the viscosities of some liquids (ii)-(vii) using liquid (i) as a reference liquid. Then, the nominal value for the viscosity of liquid (i) in Table I is used as an priori reference; $\eta_{\text {ref }}=141 \mathrm{~Pa}$ s. The experimentally obtained $G_{c r}=2.30$ and $\omega /(2 \pi)=4.21 \mathrm{~Hz}$ are also used as reference values of critical gain $G_{c r-r e f}$ and response frequency $\omega_{\text {ref }} f(2 \pi)$. Then, by taking into account Eq. (23), the viscosities $\eta$ of liquids (ii)-(vii) can be estimated by

$$
\eta_{\text {ident }}=\left(\frac{G_{c r}}{G_{c r-r e f}}\right)^{2} \frac{\rho_{r e f} \omega_{r e f}}{\rho \omega} \eta_{r e f},
$$

where $\omega$ and $G_{c r}$ are experimentally obtained for each liquid sample and for $\rho$, the known values in Table I are used. As seen from Eq. (24), the values of the constant gains of the circuit, $G_{S}, G_{P}$, and $G_{A}$, are not needed in the estimation of $\eta_{\text {ident }}$. Figure 6 shows the relationship between the experimentally determined values of the liquids $\eta_{\text {ident }}$ and their nominal values $\eta$ listed in Table I. The measurement for each liquid sample is performed 20 times; the plot points and vertical bar denote average $\eta_{a}$ and one standard deviation $\eta_{\sigma}$ from the average. If for a given temperature the data point falls on the line of gradient unity, then the average of the estimated value at the temperature is in complete agreement with the nominal value at that temperature. As seen from Table I, the deviations of the average of the experimentally
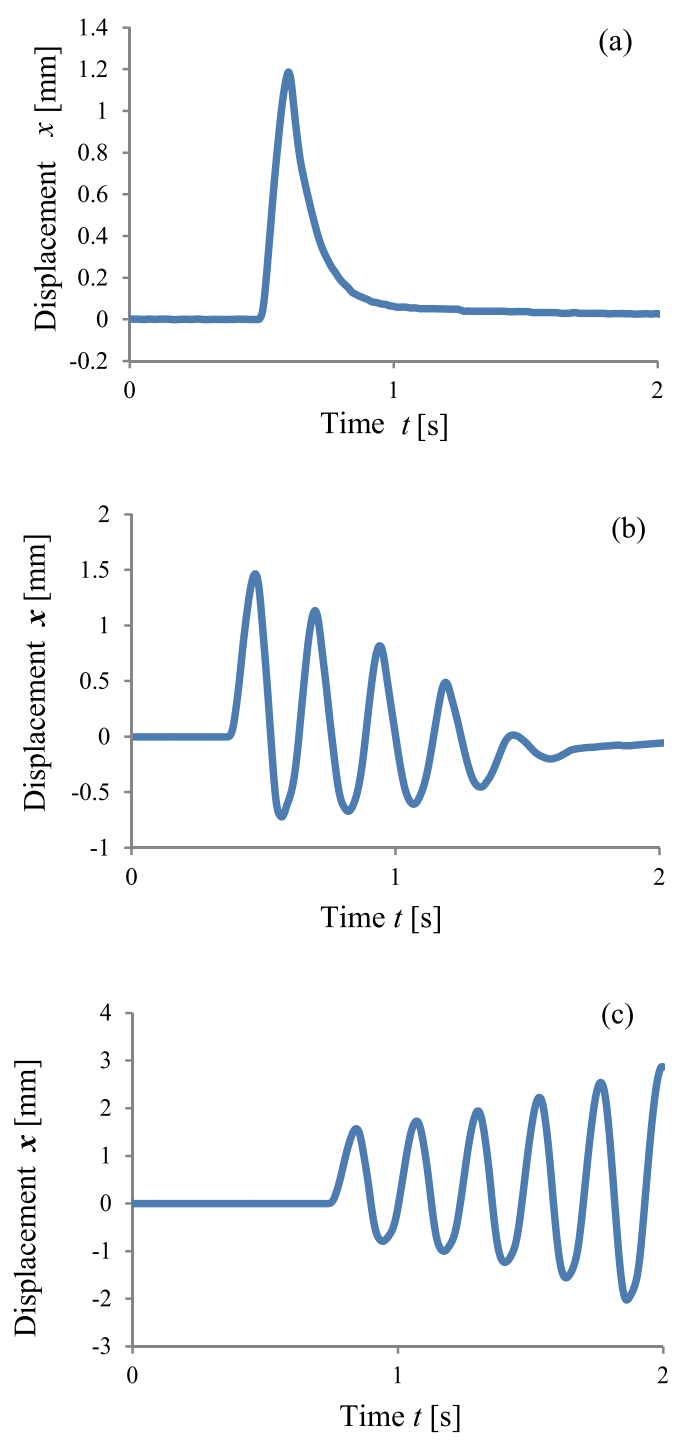

FIG. 5. Experimental time histories of the disk as a function of feedback gain $G$ : (a) without feedback $(G=0)$; due to high viscosity, the free oscillation is overdamped. (b) With feedback gain below the critical value $\left(G<G_{c r}\right)$; because the feedback control force affords compensation for energy losses through dissipation under high viscosity, the response is oscillatory, but because of insufficient compensation, the response decays with time. (c) With feedback gain above the critical value $\left(G>G_{c r}\right)$; a sufficient feedback force provides compensation for energy losses due to dissipation and produces a growing oscillation by self-excitation.

estimated values from the nominal values for liquids (ii)-(vi) are below $10 \%$. We conclude that the proposed method has also quantitative validity as a high-viscosity measurement method.

Finally, we discuss the influence of the additional fluid force caused from edge effects, which were neglected in Eq. (6). Similar to the case of the half plane, ${ }^{19}$ and also in the case of the disk, the additional fluid friction force caused by an edge depends on the depth of penetration. A modified area $S^{\prime}$ reflecting the effect should be used instead of the real area of the plate $S$ in Eq. (21). In this study, because a reference liquid is used, then if the modified area $S^{\prime}$ of the measurement liquid is approximately equal to the modified area $S_{r e f}^{\prime}$ of the reference liquid, the effective modification, i.e., the additional friction force, does not appear in Eq. (24). 


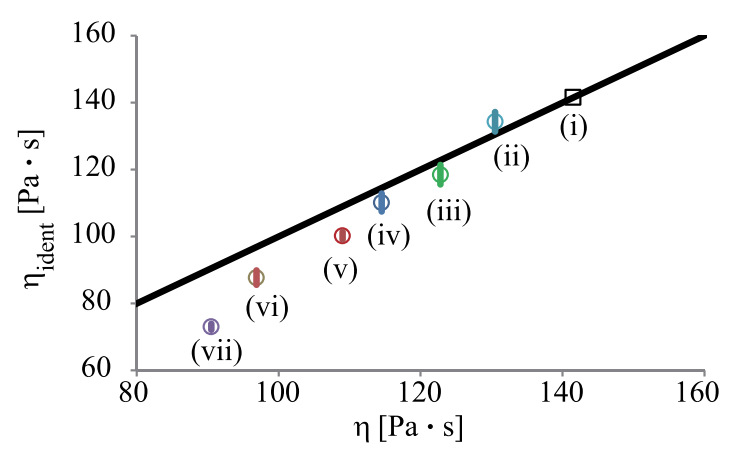

FIG. 6. Measurement results for the liquid samples listed in Table I; liquid (i) is used for calibration. The abscissa and ordinate denote, respectively, the nominal value shown in Table I and the experimentally obtained value using the proposed method. The measurement for each sample liquid is performed 20 times; plot points and vertical bars denote average and one standard deviation from the average. When data points fall on the straight line of gradient 1 , the average of the estimated viscosity is in complete agreement with the nominal value.

Nevertheless, a significant deviation between $S^{\prime}$ and $S_{\text {ref }}^{\prime}$ may cause inaccuracy in Eq. (24). Using $S^{\prime}$ and $S_{r e f}^{\prime}$, Eq. (24) can be rewritten from Eq. (23) as

$$
\eta_{i d e n t}=\left(\frac{G_{c r}}{G_{c r-r e f}}\right)^{2} \frac{\rho_{r e f} \omega_{r e f}}{\rho \omega}\left(\frac{S_{r e f}^{\prime}}{S^{\prime}}\right)^{2} \eta_{r e f}
$$

$S^{\prime}$ may decrease as the kinetic viscosity decreases, as mentioned for the half-plane in Ref. 19. Therefore, the frictional force from edge effects, which were neglected in the estimation by Eq. (24), can be regarded as one of the reasons for the underestimation of liquid viscosities in Fig. 6.

\section{CONCLUSIONS}

In conclusion, we have proposed using the self-excited oscillations for high-viscosity measurements. Unlike the conventional methods based on external or forced excitation, the frequency response curve, in addition to the high $Q$ factor, is unnecessary in the proposed method in determining high-valued viscosities of liquid samples. A macroscale oscillator as a spring-mass system is used in the experimental investigation. Miniaturized oscillators of micro- or nanoscale cantilevers can also be self-excited in a straightforward application of the proposed method. They are expected to enable not only localized monitoring of changes with time of high-valued viscosities but also spatial measurements of such viscosities using a distributed arrangement of devices.

\section{ACKNOWLEDGMENTS}

This work was partially supported by a Grant-in-Aid for Scientific Research C (Grant No. 25420195) from the Japan Society for the Promotion of Science (JSPS).

${ }^{1}$ J. G. Woodward, J. Acoust. Soc. Am. 25, 147-151 (1953).

${ }^{2}$ J. G. Woodward, J. Colloid Interface Sci. 6, 481-491 (1951).

${ }^{3}$ A. R. H. Goodwin, C. V. Jakeways, and M. M. Lara, J. Chem. Eng. Data 53, 1436-1443 (2008).

${ }^{4}$ I. Puchades and L. Fuller, J. Microelectromech. Syst. 20, 601-608 (2011).

${ }^{5}$ S. Kim, K. Kihm, and T. Thundat, Exp. Fluids 48, 721-736 (2010).

${ }^{6}$ A. I. Fedorchenko, I. Stachiv, and W. C. Wang, Flow Meas. Instrum. 32, 84-89 (2013).

${ }^{7}$ I. Lee, K. Park, and J. Lee, Rev. Sci. Instrum. 83, 116106 (2012).

${ }^{8}$ J. E. Sader, J. Appl. Phys. 84, 64-76 (1998).

${ }^{9}$ A. Agoston, F. Keplinger, and B. Jakoby, Sens. Actuators, A: Phys. 123-124, 82-86 (2005).

${ }^{10}$ B. Jakoby, F. Luclum, E. K. Reichel, and T. V. Brunnmaier, IEEE Trans. Ultrason. Ferroelectr. Freq. Control 57, 111-120 (2010).

${ }^{11}$ A. I. Fedorchenko, I. Stachiv, J. H. Ho, A. B. Wang, and W. C. Wang, Sens. Actuators, A. 147, 498-503 (2008).

${ }^{12}$ W. Wang and C. Liu, Rev. Sci. Instrum. 84, 075007 (2013).

${ }^{13}$ H. Sone, Y. Fujinuma, and S. Hosaka, Jpn. J. Appl. Phys. 43, Part 1, 3648-3651 (2004).

${ }^{14}$ H. Yabuno, M. Kuroda, T. Someya, K. Mishimura, K. Hayashi, and K. Ashida, Jpn. J. Appl. Phys. 50, 076601 (2011).

${ }^{15}$ K. Moran, C. Burgener, K. Turner, and S. Shaw, NOLTA 4, 198-224 (2013).

${ }^{16}$ H. Yabuno, Y. Seo, and M. Kuroda, Appl. Phys. Lett. 103, 063104 (2013).

${ }^{17}$ J. E. Sader, T. P. Burg, and S. R. Manalis, J. Fluid Mech. 650, 215-250 (2010).

${ }^{18}$ A. H. Nayfeh and D. T. Mook, Nonlinear Oscillations (WileyInterscience, New York, 1979).

${ }^{19}$ L. D. Landau and E. M. Lifshitz, Fluid Mechanics (ButterworthHeinemann, Oxford, 1999), pp. 83-85.

${ }^{20}$ A. H. Nayfeh, Perturbation Methods (Wiley-Interscience, New York, 1973).

${ }^{21}$ J. M. T. Thompson and H. B. Stewart, Nonlinear Dynamics and Chaos (Wiley, Chichester, 2002).

${ }^{22}$ H. Yabuno, Y. Kunitho, and T. Kashimura, J. Vib. Acoust. 130, 041008 (2008). 